\title{
Cosmic-ray world with gamma-ray astronomy: a wealth on information, an even more open issue
}

\author{
Martina Cardillo ${ }^{1, a}$ \\ ${ }^{1}$ INAF - Osservatorio astrofisico di Arcetri, Largo Enrico Fermi 5, 50142, Florence
}

\begin{abstract}
Since from their discovery in 1912, Cosmic-Rays (CRs) are one of the most debated issues of the high energy astrophysics. Their origin is still a fundamental problem and is the subject of very intense research. Until now, the best candidate sources of Galactic CR component are Supernova Remnants (SNRs) but final proof for the origin of CRs up to the knee can only be obtained through two fundamental signatures, the detection of a clear gamma-ray signature of $\pi^{0}$ decay in Galactic sources and the identification of sources emitting a photon spectrum up to PeV energies. Both indications are quite difficult to obtain. The two gamma-ray satellites, AGILE and Fermi, together with ground telescopes operating in the TeV energy range (HESS, VERITAS and MAGIC), collected a great amount of data from SNRs. In spite of the recent discovery of the neutral pion spectral signature in the SNR W44 spectrum by AGILE (and confirmed by Fermi-LAT), all gamma-ray data collected at $\mathrm{GeV}$ and $\mathrm{TeV}$ energies for several young and middle-aged SNRs provide interesting challenges to current theoretical models. The emerging view from gamma-ray and particle detection is intriguing and lead to revisit the CR-SNR paradigm, considering also the contribution of other kind of sources.
\end{abstract}

\section{Introduction}

Cosmic-rays (CRs) are highly energetic particles (with kinetic energies up to $E=10^{20} \mathrm{eV}$ ) mainly composed by protons and nuclei with a small percentage of electrons $(1 \%)$. Since from their discovery Cosmic-Rays are one of the most debated issues of the high energy astrophysics. Their origin is still a fundamental problem and is the subject of very intense research $[31,53,61]$, [for recent reviews, see 23, 67]. Their acceleration is described by diffusive shock acceleration mechanisms (DSA) $[29,44,50$, 53]. that provides a primary proton power-law distribution index $p \sim 2$, harder if non-linear effects are considered $[29,32,84]$. Because of the interaction with magnetic field, CR particle trajectories are curved and randomized; consequently, their detection on Earth is uncorrelated with the one of their sources. For this reason, electromagnetic radiation in the gamma-ray energy band is a main source of information about CRs and their sources. Contribution to the galactic gamma-ray emission is due both to protons, through the decay of $\pi^{0}$ due to pp-interactions, and electrons through different radiation mechanisms (synchrotron, non-thermal Bremsstrahlung and inverse Compton). Electromagnetic radiation from these energy loss processes can be measured and analyzed from Earth and we will see that, in order to distinguish electron from proton components, a comparative study in the whole electromagnetic spectrum is fundamental.

Focusing on CRs produced in our Galaxy (energies up to the "knee", $E=10^{15} \mathrm{eV}$ ), strong shocks in Supernova
Remnants (SNRs) are considered the most probable CR sources [e.g., 61], [recent review in 107]. SNRs are the envelopes of very massive stars ejected away during their explosion like Supernovae. They are extended sources quite near to us and, for these features, their multiwavelength study is much easier than in other objects. Indeed, we have a lot of collecting data in every frequency range of a large amount of SNRs; however, the final proof for the origin of CRs up to the knee can only be obtained through two fundamental signatures. The first one is the detection of a clear gamma-ray signature of $\pi^{0}$ decay in Galactic sources; the second one is the identification of sources emitting a photon spectrum up to $\mathrm{PeV}\left(10^{15} \mathrm{eV}\right)$ energies. Both indications are quite difficult to obtain. The "Pevatron" sources are notoriously difficult to find [for a review, see 23], and the neutral pion decay signature is not easy to identify because of the possible contribution from cospatial leptonic emission. Hadronic and leptonic components can in principle be distinguished in the $50-200 \mathrm{MeV}$ energy band, where they are expected to show different behaviors. At the light of this, it is clear that gamma-ray astronomy has a fundamental role in this context. The techniques for detection of gamma-ray radiation are different for different energy ranges. Gamma-ray detectors in space observatories are used for the low-energy range $(\mathrm{MeV}$ $\mathrm{GeV}$ ) where we can directly measure the gamma-ray flux because of its high value. For higher energies $(\mathrm{GeV}-\mathrm{TeV})$, instead, the gamma-ray flux is very faint and direct measurements are challenging. We need ground-based experiments that use the Cherenkov radiation in order to detect higher flux. Only with the development of both tech-

\footnotetext{
a e-mail: martina@arcetri.astro.it
} 
niques, we can have a complete and detailed information about CR physics. Over the last five years, AGILE and Fermi gamma-ray satellites, together with ground telescopes operating in the TeV energy range, HESS, VERITAS and MAGIC, collected a great amount of data from SNRs [3-9, 11-13, 18, 20, 21, 25, 63, 64, 69, 75, 82, 102] providing important information and challenging theoretical models. From one hand, for the first time AGILE detected the "pion bump" in the SNR W44 [41, 65], then confirmed by Fermi-LAT that found it also in another SNR, IC443 [17]. On the other hand, both these sources, classified as middle aged SNRs ( $t>1000 \mathrm{yrs}$ ) have a very steep high energy gamma-ray index, $p \geq 3$, very challenging because in contradiction with Diffusive Shock Acceleration Models (DSA) that provide an injection index $p \leq 2$, steepened by propagation effects at $p \sim 2.7$ [28, 32, 84]. Most of the young and middle-aged SNRs detected until now show, apparently, a spectrum steeper than the one expected from linear and non-linear DSA. Moreover, any SNR shows chances to reach PeV energies.

Clearly, faced with these results, theoretical models of CR acceleration and propagation need to be revised and new hypothesis about possible Galactic sources are proposed (e.g., Superbubbles). In spite of the great amount of data from SNRs emission, the understanding of CR acceleration and propagation processes is all but complete.

\section{Cosmic-Rays and SNRs}

SNRs were proposed as CR sources for the first time in 1934, in a conference proceeding of Baade and Zwicky [26], and in 1954, Vitalii Ginzburg elaborated on the SNR hypothesis with energetic considerations [60-62]. In order to experimentally test the SNR hypothesis validity, there are some fundamental requirements to be observed: acceleration efficiency of about $10-20 \%$, spectral behavior reproduction of all the elements, correct description of chemical abundances, good description of multifrequency observations, anisotropy induced by SNR spatial distribution compatible with observations. In light of these requirements, even before the coming of gamma-ray satellites and instruments, there are some important arguments supporting SNR hypothesis [19]. First, the power required in order to explain the CR flow is only a few percent of the mechanical energy released by SN explosions. Then, diffusive shock acceleration is the only model of particle acceleration that, so far, allows quantitative calculations. Moreover, the presence of high magnetic fields are a fundamental condition for CR acceleration with energy beyond $E=100 \mathrm{TeV}$. Rapid variability of X-ray emission is a strong evidence for magnetic field amplification around SN shell; indeed, only synchrotron radiation from ultrarelativistic electrons accelerated at shock fronts can satisfy the right conditions and explain this feature [103]. Theoretical considerations indicate that SNRs can accelerate the highest energy CRs during free expansion and SedovTaylor phases. In the free expansion phase, $E_{m}$ increases with time (considering B constant) and a particle can reach $E \sim 10^{17} \mathrm{eV}[30,67]$; in the Sedov-Taylor phase, shock slows down and magnetic field intensity decreases, leading to a faster decrease of $E_{\text {max }}$. This can explain the fact that older SNRs are not prominent sources of gamma-ray and also the absence of a TeV $\gamma$-ray signal from many SNRs [93].

\subsection{SNRs: Multiwavelength analysis importance}

In order to have a complete understanding of the role of SNRs in the CR acceleration, we need to know these sources from every point of view: morphology, expansion parameters, spectral behavior, interaction with the environment (InterStellar Medium, Molecular clouds). Consequently, every frequency of the electromagnetic spectrum can give us fundamental information. The radio energy band gives us information about the morphology and dimension of the SNR shell through the synchrotron emission by $\mathrm{GeV}$ electrons due to the presence of interstellar magnetic field, modified by shock wave impact. Moreover, from synchrotron photon index we can deduce the primary electron distribution behavior and, consequently, shock wave properties, especially if the radio emission is correlated with emission in other energy bands, e.g. CO [43]. Moreover, wee can obtain information about the polarization of the sources and, consequently, about the magnetic field [e.g. 67, 95], whose amplification has a fundamental role in the acceleration mechanisms [78, 79]. Gamma-ray emission from a SNR is enhanced by the presence of a dense target, such as a molecular cloud; indeed, in the most of gamma-ray observed SNRs, molecular cloud complexes are detected (see Table 1) and this correspondence could be a signature of CR acceleration. The most important tracers of the ISM neutral component are HI observations at $21 \mathrm{~cm}$ and $\mathrm{CO}$ observations at $2.6 \mathrm{~mm}$ that indicate the presence of atomic and molecular gas, respectively. Infrared emission provides information about both SNae and dust in their environment because the SN light is absorbed by dust and emitted at longer wavelengths. The most important characteristic of the dust is that it has no chemical issues, differently from molecules. Consequently, information in this energy band, if available, is more reliable. IR spectral lines confirm the presence of a shock and allow us to understand also its nature [94, and therein]. In the optical energy band, the most important role is of the Balmer lines, a very important source of information. The collisionless nature of the SNR shocks implies that, if the surrounding medium is partially ionized, shock formation involves only the ionized plasma components. Neutrals, indeed, are affected by the shock just by charge-exchange process, that is an electron exchange between an ion and a neutral. A shock where the charge-exchange process takes-place is called a "Balmer dominated shock" because this mechanism leads to Balmer line emission affected by the eventual presence of CRs [36, and therein]. This effect was observed in different SNRs as SN 1006 and Tycho [81, 99]. In the X-ray band we have two kinds of emission: thermal and non-thermal. Thermal X-ray emission from SNRs can be mostly seen in young SNRs 
because it is due to hot post-shock electrons heated by SNR shock. Consequently, non detection of thermal $\mathrm{X}$-ray emission from a remnant could mean non efficient CR acceleration [see, for instance, 100, for the SNR RX J1713]. In the so called mixed-morphology SNRs, thermal X-ray emission comes from the center of the shell [the SNR W44 is an example, see 98] and it is no clear what is the right mechanism. Non-thermal $\mathrm{X}$-ray radiation, instead, is produced through synchrotron emission of ultra-relativistic electrons and it is dominated by the downstream shock, where the magnetic field is higher. The emission region is determined by diffusion and is proportional to the magnetic field. Consequently, there is a strong link between magnetic field value and the emission region width [33, 108, and therein]: narrower is the region stronger is the magnetic field, probably amplified [91, 107, and therein]. It is clear that most of the information comes from the correlation between all the wavelengths. For instance, a correspondence between $\mathrm{CO}$ emission and cold dust emission in the FIR band could imply the presence of a SNR/MC interaction [74]. The same process is indicated also by the correlation with optical and X-ray filaments and correlation between $\mathrm{H}_{2}$, CO and radio filaments emission [94, and therein]. However, without the presence of gamma-ray emission analysis, we can not have a complete picture of the role of a SNR in the CR context.

\section{Gamma-ray from MeV to TeV: what are we looking for?}

We have seen that the gamma-ray energy band is the most important one for understanding $\mathrm{CR}$ acceleration and propagation. The advent of gamma-ray satellites (AGILE [101] and Fermi-LAT [2]) and Cerenkov telescopes (HESS [70], MAGIC [47], VERITAS [111]) allowed to analyze the correctness of linear and non-linear DSA models and to develop new more structured theories. In Table 1, we consider the most important SNRs detected so far in the gamma-ray energy band. Most of them emit in both $\mathrm{GeV}$ and $\mathrm{TeV}$ energies but with different fluxes. GeV gammaray emission from young SNRs is less than the middleaged SNR one and, usually, they strongly emit in the TeV energy band. This is attributed to energy dependent particle diffusion and escape. Moreover, propagation effects are the explanation of the strong $\mathrm{TeV}$ emission from two middle-aged SNRs, IC443 [11, 24] and W28 [21]. VHE CRs that have already left the remnants, indeed, interact with molecular clouds near the source and then we can see their emission. For the same reason we can find lowenergy particles (MeV-GeV) inside a middle-aged SNR shell. In spite of several SNRs observed, testing the SNR hypothesis for CR acceleration is challenging. A first confirmation could be dut to the detection of Balmer line emission in correspondence of the shock [68, 86, and therein]: neutrals in the upstream go through the non-collisional shock and interact by charge-exchange process with downstream ions, producing Balmer lines. Their width is modi- fied according to particle characteristic and so they can be an important proof for CR acceleration. However, this is only an indirect proof. Two direct signatures of CR acceleration by SNRs are in the gamma-ray emission regime: the pion bump and the Pevatrons.

\subsection{A first signature: the "pion bump"}

Accelerated CR protons can interact with other target protons producing, among other particles, also neutral pions that decay in two gamma-ray photons. Their spectrum has a cut-off at $E \leq 67 \mathrm{MeV}$ and below this energy it steepens. This spectral feature is one of the signatures that can prove the hadronic origin of the detected gamma-ray emission. This is a direct proof because the photon spectral index is the same of the primary protons and, consequently, we can obtain information about primary proton population. The problem is that also ultra relativistic electrons emit in the same energy band of protons through Bremsstrahlung and IC processes (see Fig. 1).

In most SNRs observed until now, both young $(t<1000$

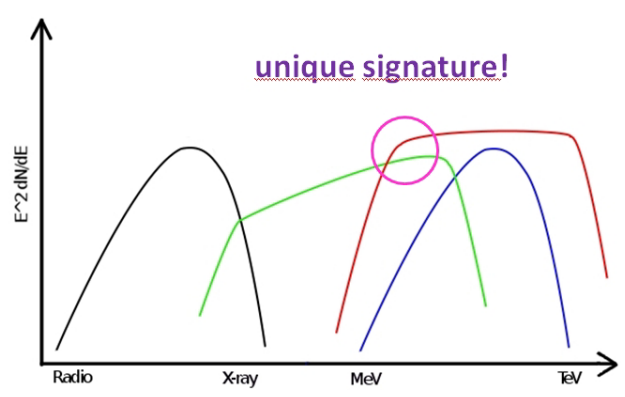

Figure 1. Scheme of the SED produced by electrons and protons inside a SNR. Electron synchrotron (black), Bremsstrahlung (green), IC (blue) emission and photon emission from pp interactions (red). It is clear the overlapping of electron and proton processes in the gamma-ray band. The magenta circle stress the energy range in which we could distinguish the two components.

yrs) and middle-aged ( $t>1000$ yrs), gamma-ray emission can be fitted with a model dominated by hadronic pion emission. In several cases we can rule out leptonic emission mechanisms but, from Fig. 1, it is clear that the most solid method for distinguishing hadronic from leptonic contribution, is to analyze low-energy spectral data. For $E \sim 200 \mathrm{MeV}$, leptonic and hadronic emissions have different behaviors and we can distinguish the two components (Fig. 1) and so, we need data below $200 \mathrm{MeV}$. However, in this energy band, observations are very challenging because of the high background and multiple electron scattering, as well as the PSF and sensitivity of gamma-ray satellites (see Fig. 2).

Something new was provided with the very bright SNR W44. This source was detected in the gamma-ray band for the first time by Fermi-LAT [8], that, however, provided spectrum only above $E=200 \mathrm{MeV}$. Consequently, it was not possible to rule out leptonic models. One year later, because of the brightness of W44 and 
Table 1. Most important SNRs detected in the gamma-ray band

\begin{tabular}{lcccc}
\hline SNR (l,b) & AGILE & Fermi-LAT & MC detect. & TeV \\
\hline CAS A (G111.7-2.1) & no & yes & yes & Veritas, MAGIC \\
Tycho (G120.1+1.4) & no & yes & yes & Veritas \\
SN 1006 (G327.6+14.6) & no & no & - & HESS \\
RXJ1713 (G347.3-0.5) & no & yes & yes & HESS \\
W49B (G43.3-0.2) & no & yes & yes & HESS \\
Puppis A (G260-3.4) & no & yes & no & - \\
$\gamma$-Cygni (G78.2+2.1) & yes & no & yes(?) & Veritas \\
W44 (G34.7-0.4) & yes & yes & yes & - \\
W51 (G49.2-0.7) & no & yes & yes & MAGIC \\
IC443 (G189.1+3) & yes & yes & yes & Veritas, MAGIC \\
W28 (G6.71-0.05) & yes & yes & yes & HESS \\
\hline
\end{tabular}

the ability to provide an energy spectrum starting at 50 $\mathrm{MeV}$ for bright objects, we analyzed AGILE data from the SNR W44, obtaining a spectrum, for the first time, with data below $200 \mathrm{MeV}$ and showing the low-energy steepening peculiar to neutral pion gamma-ray emission (see Section 4). During the last years, Fermi-LAT improved its software [16], and obtained data at low-energies for the SNR W44, confirming the pion bump discovered by AGILE and obtaining low-energy data also for the SNR IC443 [17]. Also in this case the spectrum shows a low-energy steepening, but until now there is not a deep multiwavelength study of this source, such as in the case of SNR $\mathrm{W} 44$, that allows rule out a possible fit with leptonic emission processes.
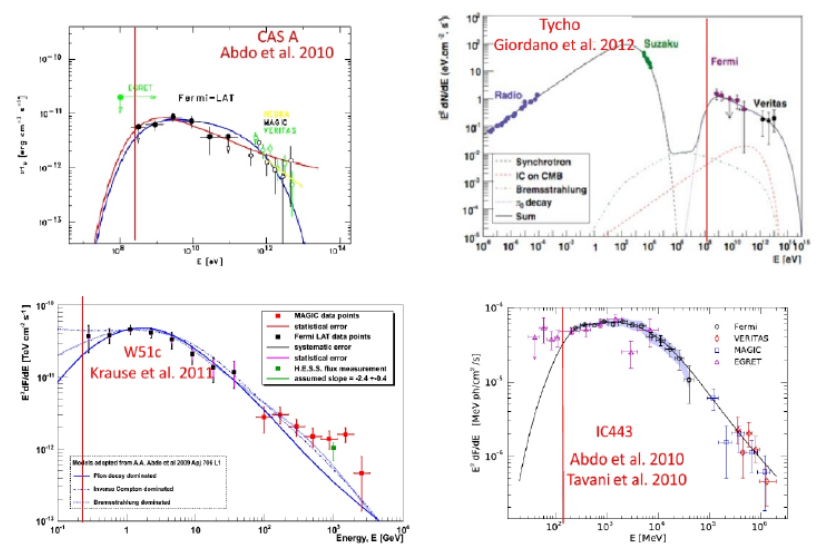

Figure 2. Gamma-ray spectra of four different SNRs, two young and two middle-aged: Upper left: Cas A [4], Upper right: Tycho [63], Bottom left: W51c [25],Bottom right: IC443 [5]. The red lines stress the lack of experimental data below $E=200$ $\mathrm{MeV}$.

\section{The case of the SNR RX J1713.7-3946}

One example of the complexity of the problem is the young SNR RX J1713.7-3946 (age 1600 yrs), which attracted considerable attention because of its intense $\mathrm{TeV}$ emission, interpreted of hadronic origin [20]. The last Fermi-LAT data on the source [9], however, show a very hard spectrum in the $10-100 \mathrm{GeV}$ range that can be con- sistent with IC leptonic emission or with hadronic emission with an unusually hard proton spectrum, $p \sim 1.7[22$, and therein]. Moreover, in [52] was shown that, if there were accelerated protons, there should be Coulomb collision with electrons; consequently, electrons are heatedup and produce some detectable emission lines. The nondetection of this signature implies a non hadronic origin of the gamma radiation. However, a recent analysis done by [56] presents a good spatial correspondence between the TeV gamma-rays and the surrounding HI gas (in addition to molecular hydrogen traced by $\mathrm{CO}$ ) then strengthening the hadronic scenario. In spite of the low average density in the SNR shell, hadronic gamma-ray emission could be explained by CR interaction with dense gas condensations embedded in the shell. In this way, the challenging low-energy emission lack in the spectrum could be due to the slow diffusion that prevents the penetration of the cloud by low-energy particles [9]. Moreover, considering clumpiness of the ISM in the SNR surroundings rather than a homogeneous density distribution, lack of the expected thermal X-ray emission (that was one of the most important arguments against hadronic scenario) can be easily explained $[56,73]$. The discussion about this interesting SNR is still open.

\subsubsection{A second signature: Pevatrons}

Another way to confirm the hadronic origin of gamma-ray emission from SNRs would be the detection of emission up to $E \sim 100 \mathrm{TeV}$ and beyond. Independently of their origin, the main contributors to galactic CRs should operate, at least during some stages of their lifetime, as 'PeVatrons' $[22,57]$ that accelerate particles up to knee energies. Indeed, electrons cannot be accelerated up to these energies because of strong synchrotron losses and because the IC component is suppressed by Klein-Nishina effects. Consequently, gamma-ray emission in this energy range can have only a hadronic origin [22, 57]. High energy particles are not expected by middle-aged and old SNRs because their escape time is smaller than the SNR age. Therefore, young SNRs are the only chance to detect PeV gamma-ray emission. 


\section{A breakthrough: The supernova remnant W44}

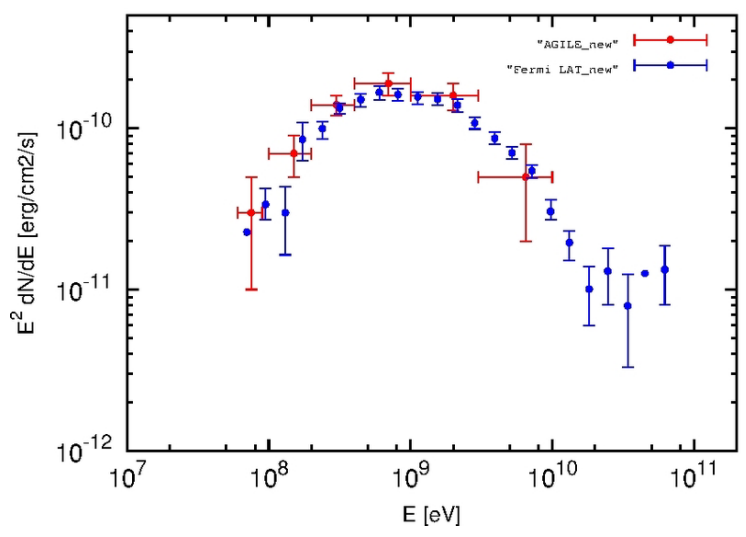

Figure 3. AGILE new gamma-ray spectrum of SNR W44 [41] superimposed with the Fermi-LAT data from [17].

The SNR W44 is a middle-aged $(\sim 20,000$ yrs old $)$ SNR located in the Galactic Plane $(l, b)=(34.7,-0.4)$ at a distance $d \sim 3.1 \mathrm{kpc}[45,112]$. Multiwavelength observations revealed interesting features. In the radio band, W44 shows a quasi-elliptical shell [42, and references therein]; the radio shell asymmetry is probably due to expansion in an inhomogeneous ISM. In the northwest side of the remnant, which correlates with a peak of the radio emission, there is bright [SII] emission characteristic of shockexcited radiative filaments [59]. In the southeast side, instead, there is a molecular cloud (MC) complex embedded in the SNR shell that interacts with the source $[96,113]$. The $\mathrm{OH}$ maser $(1720 \mathrm{MHz})$ emission detected in correspondence with the SNR/MC region, confirm their interaction [46, 71]. In [112] the discovery of the radio pulsar PSR B1853+01 is reported, which is located in the south part of the remnant and surrounded by a cometaryshaped pulsar wind nebula (PWN) [55]. This system, however, does not appear to be correlated with the detected gamma-ray emission. The X-ray observations of W44 by the Einstein Observatory [110] showed centrally peaked emission, which is later confirmed by Chandra data [98]. The SNR W44 is well studied also in the gamma-ray band. In [8], a GeV morphology well correlated with the radio emission is showed, together with a steep photon spectrum (index near 3) that, however, has a low-energy threshold of $200 \mathrm{MeV}$, limiting the chance to identify a neutral pion signature. The relatively large gamma-ray brightness of W44 and the good spectral capability of AGILE near $100 \mathrm{MeV}[105,106]$ have stimulated a thorough investigation of this supernova remnant with the AGILE data. The AGILE gamma-ray spectrum in the range of $50 \mathrm{MeV}$ to $10 \mathrm{GeV}$ confirms the high-energy steep slope up to 10 $\mathrm{GeV}$ and, remarkably, identifies a spectral decrease below $200 \mathrm{MeV}$ for the first time, as expected from neutral pion decay [65]. The low-energy spectral behavior seen by AGILE was recently confirmed by the Fermi-LAT team that revisited the gamma-ray emission from W44
[17]. Their best hadronic model with an assumed surrounding medium density $n \sim 100 \mathrm{~cm}^{-3}$ is based on a smoothed broken power-law hadronic distribution with a break energy $E_{b r}=22 \mathrm{GeV}$ and indices $p_{1}=2.36$ for $E<E_{b r}$ and $p_{2}=3.5$ for $E>E_{b r}$. A new analysis of AGILE data with a revised assessment of the W44 surrounding environment, based on new $\mathrm{CO}$ data obtained from the NANTEN2 telescope that provide an average density $n \sim 200 \mathrm{~cm}^{-3}$ [114], confirms a broken power-law proton distribution characterized by an index $p_{1}=2.2 \pm 0.1$ (for $E<E_{b r}$ ), $p_{2}=3.2 \pm 0.1$ (for $E>E_{b r}$ ), and an energy break $E_{b r}^{p}=20 \mathrm{GeV}$. The leptonic contribution to this model is given by a simple power-law for the electrons with $p^{\prime}=1.74$, and $E_{c}^{e}=12 \mathrm{GeV}$ (see Fig. 4). This model provides a proton energy $W^{p}=5 \times 10^{49} \mathrm{erg}$ and requires an average magnetic field in the emission region, $B=210$ $\mu \mathrm{G}[41]$.

Summarizing, the gamma-ray analysis, compared with
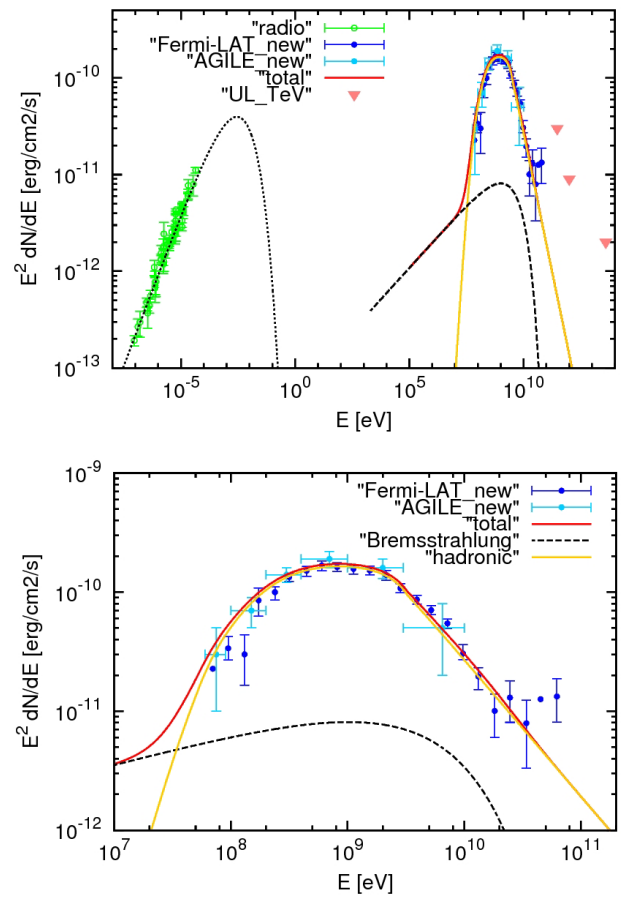

Figure 4. Hadronic model of the broadband spectrum of the SNR W44 that is superimposed with radio and gamma-ray data of Fig. 3 [41]. It is a broken power-law proton distribution in momentum with index $p_{1}=2.2 \pm 0.1$ (for $E<E_{b r}$ ) and $p_{2}=3.2 \pm 0.1$ (for $E>E_{b r}$ ) where $E_{b r}^{p}=20 \mathrm{GeV}$. This model is characterized by $B=210 \mu \mathrm{G}$ and $n=300 \mathrm{~cm}^{-3}$. The black curves show the electron contribution by synchrotron (dot) and Bremsstrahlung (dashed) emissions; the IC contribution is negligible. The total gammaray emission from pion-decay and Bremsstrahlung is also shown. (Upper Panel): SED from radio to gamma-ray band. (Bottom Panel): only gamma-ray part of the spectrum.

multiwavelength one, of the SNR W44, gave us some fundamental information about this source. First of all, the SNR W44 is the first SNR clearly showing the so-called "pion bump" [17, 41, 65]. CO emission analysis determined a very high average density in the W44 shell of $n_{a v} \sim 300 \mathrm{~cm}^{-3}$ [114]. This feature was also found in 
other middle-aged SNRs, like W51c and IC443 [43, 76] and explains the high gamma-ray flux detected from these sources. Combined analysis of radio and gamma-ray data, allowed us to give an estimation of the magnetic field, $B \geq 100 \mu \mathrm{G}$ [41], substantially higher than the equipartition magnetic field [42]. In most of SNRs, magnetic field estimations give values $B \sim 10-10^{2} \mu \mathrm{G}$ that are much higher than the average diffuse galactic value [e.g., see [86] for Tycho, [76] for W51c, and [102] for IC443]. This is hardly surprising since magnetic field compression due to the shock interaction with the ISM leads to its amplification. We need to consider a non-linear scenario with a back-reaction of the accelerated particle at the shock [30]. The large value for the magnetic field in W44 may be linked to the environment density value, $n_{a v} \sim 300 \mathrm{~cm}^{-3}$ given by NANTEN2. The most challenging feature of the SNR W44 is the high energy spectral index $p_{2} \sim 3$ $[8,17,41,65]$ that is steeper than the values found in other middle-aged SNRs. Alfvèn damping in a dense environment [85] is a mechanism for explaining this behavior, but other possibilities exist [e.g., 34, 35]. This is a point requiring deeper investigations in the future.

\section{W44 and the other gamma-ray emitting SNRs}

\subsection{W44 and W28: comparison}
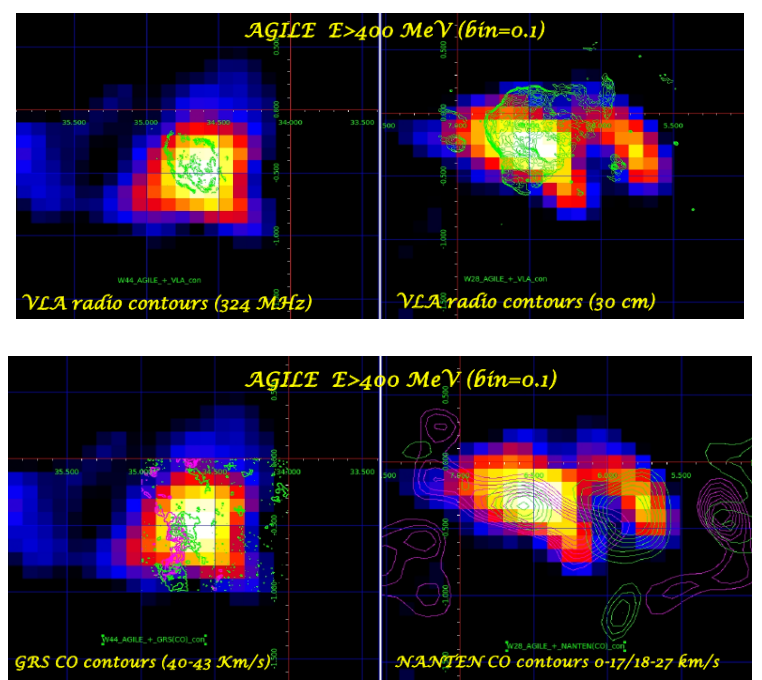

Figure 5. AGILE gamma-ray maps of the SNR W44 (left) and the SNR W28 (right) for $\mathrm{E}>400 \mathrm{MeV}$ and with binsize $=0.1^{\circ}$, in galactic coordinates. In the top panel, radio contours from VLA are overlapped; in the bottom, CO contours from GRS for W44 and from NANTEN fro W28 [40].

At the light of the SNR W44 characteristics and of the SNR/CR context, we want to present a comparison between this very important source and another important SNR detected in the gamma-ray band, both at $\mathrm{GeV}$ and TeV energies, SNR W28 [7, 21, 64]. Even W28 is a middle-aged mixed morphology SNR with dimensions of the shell radio very similar to the W44 ones. In spite of this, from Table 2 and from Fig. 5, we can see that these two remnants have some very different features that lead to a different interpretation of their gamma-ray spectrum. First of all, from the upper panel of Fig. 5, we can see that W44 gamma-ray emission has a very good correlation with its radio shell, differently by W28 where no correspondence there is between gamma-ray emission and radio shell. Observing gamma-ray/CO emission correlation (bottom panel of Fig. 5), in W44 the MC seems to be embedded in the remnant; in W28, instead, two different MCs are perfectly correlated with $\mathrm{GeV}$ and $\mathrm{TeV}$ peaks. This is confirmed also by estimated average densities of the two SNR shells (see Table 2); very small for W28 and of order of $10^{2}$ for $\mathrm{W} 44$. Moreover, no TeV emission was detected from W44, differently from W28. This could be explained by the fact that in the case of W44, gamma-ray emission comes from both MC and the SNR shell; TeV particles could be are already escaped by the remnant. W28 is older than W44 and this implies that the most part of GeV and $\mathrm{TeV}$ CR particles are escaped from the remnant. Consequently, no emission is detected correlated with the shell but only with the two MCs [40].

This difference is fundamental for their spectral behavior interpretation. We have seen that for W44 a simple linear DSA model fails because high energy spectral-index is $\sim 3$ when linear DSA model provides an index of 2.6-2.7. For this reason, we need to consider all possible non-linear mechanisms that could explain the steepening of the spectrum. In the case of W28, instead, a simple linear DSA model can easily explain its spectral behavior. In [64], we find that where is the peak of $\mathrm{GeV}$ emission there is a minimum in the $\mathrm{TeV}$ emission, and viceversa. This behavior reflects also in the spectrum; we can divided it into two components. One from the East cloud, where there is the $\mathrm{GeV}$ maximum, and the other from the West cloud complex, where is the TeV maximum [21, 64]. From [64], considering the two clouds at different distances, we can explain gamma-ray data from W28 with the simple energy dependent diffusion, with $\delta \approx 0.5$ and $D_{0}=10^{26} \mathrm{~cm}^{2} \mathrm{~s}^{-1}$ (Bohm diffusion regime).

In light of these considerations, it is clear that even in W28 the effect of some non-linear mechanisms affect the system; consequently, considering only the diffusion mechanism in order to understand its characteristic seems a oversimplification. However, the low average density of the SNR shell, and the absence of MCs embedded within it exclude most of the non-linear mechanisms considered so far.

\subsection{A more general overview}

Now, we want to give a picture of the CR/SNR context from a more general point of view. For this purpose, we need to analyze separately young and middle-aged SNRs: indeed, the first ones give information about injection and acceleration mechanism in correspondence of the SNR shell. The second ones, about particle escape, propagation and possible re-acceleration.

The importance of young SNRs is related to two funda- 
Table 2. W44 and W28 parameters

\begin{tabular}{lcccccccc}
\hline SNR (1,b) & $\begin{array}{c}\text { distance } \\
\text { kpc }\end{array}$ & $\begin{array}{c}\text { age } \\
\text { yrs }\end{array}$ & $\begin{array}{c}\text { radio } \\
\text { index }\end{array}$ & $\begin{array}{c}\text { GeV } \\
\text { index }\end{array}$ & $\begin{array}{c}\text { TeV } \\
\text { index }\end{array}$ & $\begin{array}{c}\text { n } \\
\mathrm{cm}^{-3}\end{array}$ & $\begin{array}{c}\text { B } \\
\mu G\end{array}$ & MC \\
\hline $\mathbf{W 4 4}(G 34.7-0.4)$ & 3.1 & $\sim 20,000$ & 0.37 & $2.4->3.0$ & - & $250-300$ & $\geq 100$ & yes \\
W28(G6.71-0.05) & $1.8-3.3$ & $35,000-45,000$ & 0.35 & $\sim 2.6-2.7$ & $\sim 2.5$ & 5 & $10^{2}-10^{3}$ & yes \\
\hline
\end{tabular}

mental issues. First, their spectra are not affected by propagation effect as in the case of middle-aged SNRs. Consequently, analysis of their spectral behavior allows to understand injection spectral index and physical processes that could have an impact on acceleration mechanism. On the other hand, their young age enhances the chance to detect emission from particles with energies up to $E \sim 10^{15}$ $\mathrm{eV}$, called Pevatrons, one of the direct signature for $\mathrm{CR}$ acceleration. The most studied young SNRs, so far, are Cas A [4, 12, 18], Tycho [12, 63], SN1006 [14] and RX $\mathrm{J} 1713$ [9, 20]. Gamma-ray data collected by satellites and instruments, however, put us in front of a very challenging reality. The first problem is that young SNR fluxes are quite faint in the gamma-ray energy band. This leads to the detection of a very low number of these objects; consequently, it is very difficult have some reliable conclusions about their general behavior. From theoretical considerations, young SNRs should to have a low energy flux at $\mathrm{GeV}$ energies, but a high energy flux in the TeV energy range. Even if the faintness of the detected gamma-ray flux is strongly related to the SNR distance, there is another parameter that can affect it intrinsically; this is the density value in the SNR surroundings. Both Cas A and Tycho SNRs expand into a low average density medium $[66,72]$, as well as the other young SNRs, even if in all cases are detected some MCs. If, on one side, this low average density can explain a low gamma-ray flux, on the other side, it is not so easy to explain the gamma-ray emission from these SNRs with a (most favorable) hadronic model. An explanation for this behavior is given in [39]; where the progenitor wind residual is considered as the CR target. Another explanation could be linked to proton trapping; in a Bohm-like diffusion regime the proton escape time is much greater than the time needed to cross the system lengths.

However, the most important challenge derived from experimental data is their spectral index, $p=2.3-2.4[4,63]$. Emission at the "knee" energies is expected by young SNRs because their emission is not affected by propagation effects. However, no sources were observed at $E>10$ $\mathrm{TeV}$. Consequently, steepening of young SNR spectrum implies no detection of Pevatrons; in the case of Cas A , the presence of high-energy cut-off enhances the system complexity. In the case of Cas A, there is also a cut-off at $E \sim 500 \mathrm{GeV}$ that is very challenging to be explained in young SNRs. One hypothesis is that, since Cas A is a core-collapse SNR, it is evolving inside its progenitor wind [107] where density $\rho \propto r^{-2}$; this could imply that Cas A accelerated CRs in the first phase of its evolution and the cut-off could be explained by particle escape.
Differently by young SNRs, middle-aged SNRs have spectra that are influenced by propagation effects. When we analyze their gamma-ray emission, we have to consider the modification of injection spectral index due to the diffusion. Clearly, it is more difficult to detect the first phases of the acceleration process and we expect to be difficult to detect Pevatrons because high energy particles are already escaped from these remnants. Detection at $\mathrm{TeV}$ energies is possible only in the presence of a target not embedded in the remnant, like in the SNR W28. In Fig. 6 (bottom panel), we collected all available $\mathrm{GeV}$ and $\mathrm{TeV}$ data of most of the middle-aged SNRs studied so far. The majority of these SNRs have a GeV flux quite high and are easily detectable. The lower flux of W49b and W51c is probably due to their great distances from us with respect to the other remnants). In all cases we detect MC complexes interacting with remnants and magnetic fields have large values. Puppis A seems to be a unique case. It has a low magnetic field and no MC are detected in their surroundings [51, 69]. Its GeV flux is low, even if its distance is not so large $(2 \mathrm{kpc})$, probably due to the absence of a dense target. Moreover, its spectrum has an index $\alpha \sim 2.1$, harder than all the other middle-aged SNRs and also than young SNRs. The middle-aged SNRs gamma-ray spectral indices are in a range $2.6 \leq \alpha \leq 3$, and, in the oldest SNRs, radio spectral index is harder than the one expected from modifications due to shock waves [42, 88]. This implies an electron index, not only harder than $\alpha \sim 2$, but also different from the proton one. All these SNRs seem to have similar surroundings and similar characteristics, such as high magnetic field, presence of MCs and so on. In spite of these facts, their gamma-ray spectral indices show that different physical mechanisms are at work. The comparison between W44 and W28 was an example (Section 5.1). In order to explain SNR spectral behavior different from the theoretical expectations, several physical processes were considered so far. One is the neutrals "return flux" that leads to a suppression of the Mach number and compression ratio due to the formation of a shock precursor $[36,48,87]$. Another is the formation of a CR-induced precursor due to the scattering center velocity. Lowenergy particles feel the lower compression factor at the subshock and the spectrum becomes steeper. This effect disappears at $E>$ few $\mathrm{GeV}$ because high energy particles feel the whole precursor [36]. How we said before the Alfvén Damping, due to the presence of neutrals in the SNR surroundings, leads to a suppression of scattering center velocity in a certain energy range and, consequently, of the acceleration efficiency [77, 85]. Last but not least, there is an argument linked to propagation. The 
DSA theory requires that the flatter the injection spectrum the larger must be the value of the diffusion index $\delta$ in order to explain the CR spectrum observed on Earth. A value $\delta>0.5-0.6$ provides an excessive anisotropy and, consequently, implies a steep injection index, in agreement with the observations but not with theoretical models. Physical process that could explain a lower spectral diffusion index is CR weak stochastic reacceleration that provide $D \propto E^{0.3}$ [33-35]. All these processes have solid physical reasons but most of them depend on poorly known parameters that, often, are considered separately. In a complex system like a SNR, we should consider all possible physical processes together, and their mutual interaction. Only in this way we can have a correct picture of the system.
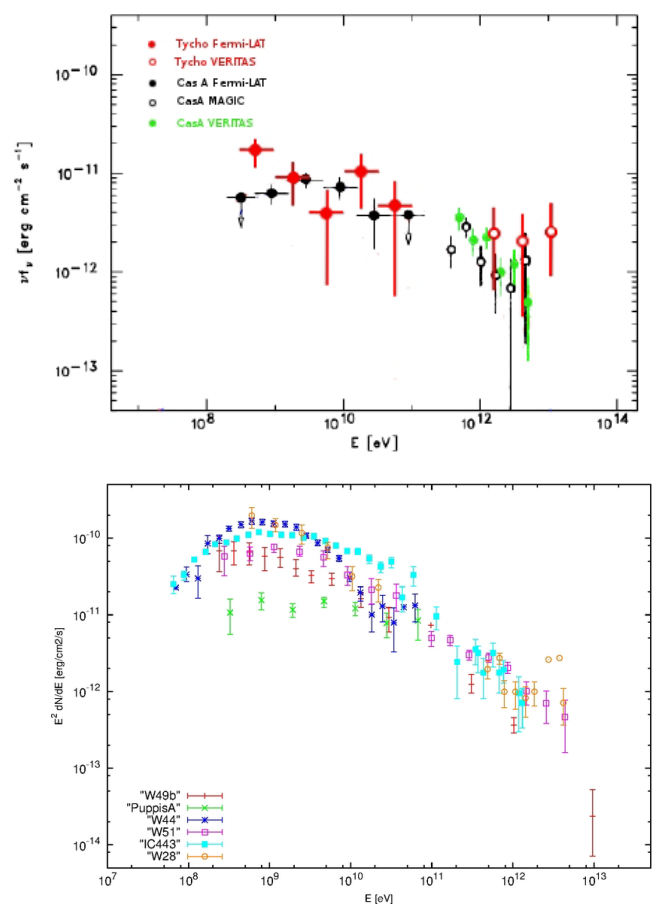

Figure 6. Upper Panel:GeV and TeV spectral points of the two young SNRs Cas A and Tycho [4, 12, 13, 63]. Bottom Panel: $\mathrm{GeV}$ and $\mathrm{TeV}$ spectral points of the most important middle-aged SNRs: W49b [6], Puppis A [69], W44 [17, 41], W51c [3, 25], IC443 [11, 17] and W28 [7, 21]

\section{The Superbubble Hypothesis}

Since the steep gamma-ray spectra detected from several SNRs indicate that the maximum particle energy, $E_{M}$, in several cases appears to be too low to produce CRs in the Pevatron range it is clear that there are new proposed candidates for CR acceleration. This is a controversial issue that is currently being studied. The best candidates so far are superbubbles, hot and rarefied cavities created by the strong winds of the OB stars [37, 38, 90, and therein]. These structures contain $\sim 94 \%$ of core-collapse SNRs that are $\sim 85 \%$ of all SNRs [83]. SNRs in OB associations represent the largest fraction of all galactic stellar ex-

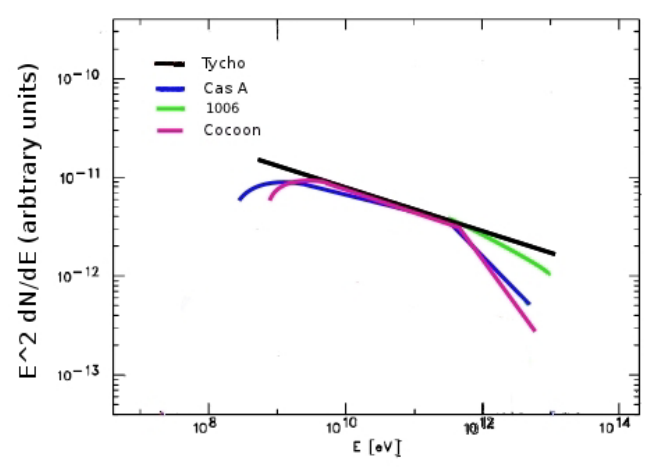

Figure 7. Normalized gamma-ray spectral behavior of the three young SNRs, Cas A (blue), Tycho (black) and SN 1006 (green), compared with the Cygnus Cocoon spectral behavior (magenta) in the hypothesis that the $\mathrm{GeV}$ and $\mathrm{TeV}$ sources coincide (see the main text).

plosions. Two are the main arguments in support of superbubble CR acceleration. The first is the different evolution of a SNR in a superbubble compared with to isolated ones; in a SB a SNR does not become radiative and so there are no radiative losses [90] but all the energy remains in the $\mathrm{SB}$, available for particle acceleration. The second one, is the generation and amplification of strong magnetic turbulence through interaction and merging of different wind bubbles and presence of clumps of higher density material [83]. Particle are accelerated at the stage of two approaching shocks [54] and, moreover, low-energy particle could be experienced multiple shock accelerations inside the SB [83]. The overall effect is a very efficient injection, a low-energy spectrum harder than that product by a single shock DSA and consequently higher EM than in an isolated SNR. Until now, there is no unambiguous evidence for superbubble CR acceleration. We notice that Fermi-LAT discovered gamma-ray diffuse emission in the Cygnus region [15], obtained from a subtraction of pointlike and extended sources such as the SNR $\gamma$-Cygni. This gamma-ray emission above $1 \mathrm{GeV}$ is spatially correlated with a photon dominated region and its morphology and its spectral behavior [15] points to a diffuse interstellar origin of this, like in a cocoon. Current superbubble models provide parameters that might explain the Cygnus Cocoon spectrum. The cocoon also includes the MILAGRO source MGRO J2031+41, an extended TeV source with a TeV emission that could not be explained only with the correlated pulsar TeV J2032+4130 [1]. A recent analysis of MILAGRO data of this TeV source [10] does not resolve the relation between it and the cocoon. Assuming that $\mathrm{GeV}$ and $\mathrm{TeV}$ emission originate from the MILAGRO source, I collected spectral data from Fermi-LAT [16] and from ARGO [27]. In Fig.7, we compare the Cygnus Cocoon spectral behavior with those of young SNRs. It is interesting to note that, if the cocoon and MGRO J2031+41 are correlated, we have a spectrum similar to the Cas A one; this seems to imply a quite steep spectrum of high energy CRs in the Cygnus Cocoon. The superbubble hypothe- 
sis needs more work to establish a clear observational evidence.

\section{Conclusions}

The CR acceleration and propagation issue is one of the most important in the field of high energy astrophysics: a century after their discovery, CR origin is not understood yet. One of the best way to approach at this problem is the analysis of the Galactic part of their spectrum, up to $E \sim 10^{15} \mathrm{eV}$, that should be more easy to study because coming from our Galaxy. The SNRs are the most probable sources of the CR galactic component and, in spite of the importance of a multiwavelegth analysis of these objects, gamma-ray astronomy has a fundamental role in verifying this hypothesis. Direct proofs of CR acceleration by SNRs, indeed, can be found only in the MeV-TeV energy band. Despite gamma-ray GeV satellites (AGILE and Fermi-LAT), and TeV instruments (HESS, MAGIC and VERITAS) collected a great amount of data on SNRs, theory and experimental data comparison led to a challenging context. Linear and non-linear theories fail in the explanation of the spectral behavior of gamma-ray produced by particle acceleration in SNRs. The same SNR W44,that is the first SNR where was observed the "pion bump" in its gamma-ray spectrum, giving the first proof of CR acceleration, is a very challenging source because of its very steep spectral index at high energies, $p \geq 3$. All SNRs studied in the gamma-ray band so far show spectral features different from theoretical predictions and there is no source with detectable gamma-ray emission at $\mathrm{PeV}$ energy. We need to collect more data in the $50-200 \mathrm{MeV}$ band, in order to find other pion signatures, and we need also more gamma-ray emitting SNRs in order to have a better statistics that enhance the chance to find $\mathrm{PeV}$ emission. The lack of low-energy data could be definitely resolved by satellites with higher sensitivity in the 10-200 MeV energy band e.g. Gamma-400 [58] or the newborn AstroGam; instead, an instrument like CTA [109] will be useful to have a better sensitivity at highest energies. Once we will have a large data statistics and a solid understanding of gammaray data, we need a deeper analysis of multiwavelength information in order to understand the discrepancy between theoretical models and data. Each band gives different information about source surroundings ( $\mathrm{CO}$, IR and optical), morphology, magnetic field (radio and X-ray) etc. Only in this way, we will be able to finally find a solution at the fascinating and challenging issue of CR origin.

\section{References}

[1] Abdo, A.A., ApJ 658, 33-36 (2007)

[2] Abdo A.A. et al. Phys. Rev. 80, 122004 (2009)

[3] Abdo, A.A. et al. ApJ, 706, L1-L6 (2009)

[4] Abdo, A.A. et al., ApJ 710, 92-97 (2010)

[5] Abdo, A.A. et al., ApJ 712, 459-468 (2010)

[6] Abdo, A.A. et al., AJ 722, 1303-13011 (2010)

[7] Abdo, A.A. et al., ApJ 718, 348-356 (2010)
[8] Abdo, A.A. et al., Science 327, 1103-1106 (2010)

[9] Abdo , A.A. et al., ApJ 734, 28-36 (2011)

[10] Abdo, A. A. et al., ApJ 753, 159-166 (2012)

[11] Acciari, V.A. et al., ApJ 698, 133-137 (2009)

[12] Acciari, V.A. et al., ApJ 714, 163-169 (2010)

[13] Acciari, V.A. et al., ApJ 730L, 720-725 (2010)

[14] Acero, F. et al., A\&A 516, 62-68 (2010)

[15] Ackermann, M. et al., Science 334, 1103-1107 (2011)

[16] Ackermann, M. et al., ApJS 203, 4-73 (2013)

[17] Ackermann, M. et al., Science 339, 807-811 (2013)

[18] Aharonian, F. A. A\&A 370, 112-120 (2001)

[19] Aharonian, F. A., Very High Energy Cosmic Gamma Radiation: a Crucial Window on the Extreme Universe, (World Scientific Publishing, River Edge, NJ, 2004) 495

[20] Aharonian, F.A. et al., A\&A 464, 235-243 (2007)

[21] Aharonian, F.A. et al., A\&A, 481, 401-410 (2008)

[22] Aharonian, F. Astrop. Phys. 43, 71-80 (2012)

[23] Aharonian, F., Astrop. Phys. 43, 71-80 (2013)

[24] Albert, J. et al., ApJ 664, 87-90 (2007)

[25] Aleksic, J. et al., A\&A 541, 13-23 (2012)

[26] Baade, W. \& Zwicky, F., Proc. of the National Academy of Sciences 20, 259-263 (1934)

[27] Bartoli, B. et al., ApJ 745, 22-26 (2012)

[28] Bell, A.R., MNRAS 182, 147-156 (1978)

[29] Bell, A.R., MNRAS 225, 615-626 (1987)

[30] Bell, A.R., MNRAS 321, 433-438 (2001)

[31] Berezinskii, V.S. et al. , Astrophysics of Cosmic Rays, (North-Holland, Amsterdam, 1990)

[32] Blasi, P., Gabici, S. \& Vannoni, G., MNRAS 361, 907-918 (2005)

[33] Blasi, P. , crpa.conf, 493-506 (2011)

[34] Blasi, P. \& Amato, E., JCAP 01, 10-39 (2012a)

[35] Blasi, P. \& Amato, E. , JCAP 01, 011 (2012b)

[36] Blasi, P., ApJ 755, 121-132 (2012c)

[37] Butt, Y.M. , Nature 460, 701-704 (2009)

[38] Bykov, A.M., SSRv 99, 317-326 (2001)

[39] Caprioli, D., arXiv1111.0116C (2011b)

[40] Cardillo, M., Giuliani, A. and Tavani, M., AIPC 1505, 221C (2012)

[41] Cardillo, M., Tavani, M., Giuliani, A. et al., A\&A 565, 74-85 (2014)

[42] Castelletti, G. et al., A\&A 471, 537-549 (2007)

[43] Castelletti, G. et al., A\&A 534, 21-34 (2011)

[44] Cesarsky, J. \& Lagage, P.O., ICRC 2, 157C (1987)

[45] Clark, D.H. \& Caswell, J.L, MNRAS 174, 267-305 (1976)

[46] Claussen, M. J., Frail, D. A. \& Goss, W. M., ApJ 489, 143-159 (1997)

[47] Cortina, J., et al. (MAGIC Collaboration), AIP Conf. Proc. 745, 730 (2005)

[48] Draine, B.T. \& McKee, C.F., A\&A 31, 373-432 (1993)

[49] Draine, B.T. et al., ApJ 264, 485-507 (1983) 
[50] Drury, L.O'C., Axford, W. I. \& Summers, D., MNRAS 198, 833-841 (1982)

[51] Dubner, G. et al., A\&A 555, 9-17 (2013)

[52] Ellison, D. C. et al., ApJ 712, 287-293 (2010)

[53] Fermi, E., Phys. Rev. 75, 1169-1174 (1949)

[54] Ferrand, G. \& Marcowith, A., A\&A 510, 101-111 (2010)

[55] Frail, D. A. et al., ApJ 464, 165-169 (1996)

[56] Fukui, Y. et al., ApJ 746, 82-99 (2012)

[57] Gabici S. et al., Astrophys. and Space Science 309, 365-371 (2007)

[58] Galper, A.M. et al., AdSpR 51, 297-300 (2013)

[59] Giacani E.B. et al., AJ 113, 1379-1390 (1997)

[60] Ginzburg, V.L., IAUS 9, 589-594 (1959)

[61] Ginzburg, V.L. \& Syrovatskii, S.I. , The Origin of Cosmic Rays, (Pergamon, Oxford, 1964)

[62] Ginzburg, V.L. , Usp. Fiz. Nauk 163, 45-50 (1963)

[63] Giordano, F. et al., ApJ 744, 2-6 (2012)

[64] Giuliani A. et al., A\&A 516, 11-13 (2010)

[65] Giuliani, A., Cardillo, M., Tavani, M. et al., ApJ 742, 30-34 (2011)

[66] Gomez, H.L., MNRAS 420, 3557-3573 (2012)

[67] Helder, E.A. et al., Space Science Reviews 173, 369431 (2012)

[68] Heng, K., PASA 27, 23-44 (2010)

[69] Hewitt, J.W. et al., ApJ 759, 89-98 (2012)

[70] Hinton, J. A., NewAR 48, 331 (2004)

[71] Hoffman, M., Goss, W. M., Brogan, C. L. and Claussen, M. J., AJ 620, 257-273 (2005)

[72] Hwang, U. \& Laming, J.M., ApJ 746, 130-147 (2012)

[73] Inoue, T., Yamazaki, R., Inutsuka, S. \& Fukui, Y., ApJ 744, 71-85 (2012)

[74] Ishihara, D., A\&A 521, 61-65 (2010)

[75] Katsuta, J., ApJ 752, 135-146 (2012)

[76] Koo, B et al., AJ textbf140, 262-265 (2010)

[77] Kulsrud, R. \& Pearce, W.P., ApJ 156, 445-469 (1969)

[78] Lagage, O. \& Cesarsky, C.J., A\&A 118, 223 (1983a)

[79] Lagage, O. \& Cesarsky, C.J., ApJ 125, 249-257 (1983b)

[80] Lande, J., ApJ 756, 5-25 (2012)

[81] Lee, J.J. et al., ApJ Letters 715, 146-149 (2010)
[82] Lemoine-Goumard, M., A\&A 545, 28-36 (2012)

[83] Lingenfelter, R.E.,AIPC 1516, 162-166 (2013)

[84] Malkov, M.A. \& Drury, O., Rep. Prog. Phys. 64, 429481 (2001)

[85] Malkov, M.A., Diamond P.H. \& Sagdeev, R.Z., Nature Com. 2, 194-198 (2011)

[86] Morlino, G. and Caprioli, D., A\&A 538, 81-95 (2012)

[87] Ohira, Y. 2012, ApJ, 758, 97-105

[88] Onic, D., Astrophysics and Space Science 346, 3-13 (2013)

[89] Osborne, J.L. \& Ptuskin, V.S., Sval. 14, 132-134 (1988)

[90] Parizot, E. et al., A\&A 424, 747-760 (2004)

[91] Parizot, E. et al., A\&A 453, 387-395 (2006)

[92] Pohl, M. et al., arXiv1411.2891P (2014)

[93] Ptuskin, V.S. \& Zirakashvili, V.N. , A\&A 429, 755765 (2005)

[94] Reach W.T. et al., AJ 618, 297-320 (2005)

[95] Reynoso, E.M. et al., AJ 145, 104-112 (2013)

[96] Rho, J. et al., AJ 430, 757-773 (1994)

[97] Seo, E.S. \& Ptuskin, V.S., ApJ 431, $705-714$ (1994)

[98] Shelton, R.L., Kuntz, K.D. and Petre, R., ApJ 611, 906-618 (2004)

[99] Sollerman, J. et al. A\&A 407, 249-257 (2003)

[100] Tanaka, T. et al., ApJ 685, 988-1004 (2008)

[101] Tavani, M. et al., A\&A 502, 995-1013 (2008)

[102] Tavani, M. et al., ApJ 710, 151-155 (2010)

[103] Uchiyama, Y. et al., Nature 449, 576-578 (2007)

[104] Uchiyama, Y. et al., ApJ 749, 35-39 (2012)

[105] Vercellone, S. et al., ApJ 676, L13-L16 (2008)

[106] Vercellone S. et al., ApJ 690, 1018-1030 (2009)

[107] Vink, J., A\&A Rev. 20, 49 (2012)

[108] Volk, H.J., Berezhko, E.G. \& Ksenofontov, L.T., A\&A 433, 229-240 (2005)

[109] Wagner, R.M., arXiv0912,3742W

[110] Watson, M. G. et al., IAUS 101, 273-280 (1983)

[111] Weekes, T. et al. (VERITAS Collaboration), Astropart. Phys. 17, 221 (2002)

[112] Wolszczan, A. et al., AJ 372, L99-L102 (1991)

[113] Wootten, H.A., ApJ 216, 440-445 (1977)

[114] Yoshiike, S., ApJ 768, 179-188 (2013) 